\title{
Arbor
}

\section{La Escuela Filosófica de Kioto como paradigma para una reflexión intercultural}

\author{
Javier del Arco Carabias
}

Arbor CLXXIX, 705 (Septiembre 2004), 229-246 pp.

Lo que caracteriza esencialmente a la escuela de Kyoto es que constituye una contribución sostenida y original del oriente lejano -Japóna la filosofía occidental desde una perspectiva característicamente oriental. La primera vez que se utilizó la denominación escuela de Kyoto fue en el Japón, en el año 1932; se trata pues de un pensamiento moderno que se genera en la Universidad Imperial de Kyoto a partir de las reflexiones y escritos de Kitaro Nishida (1870-1945), catedrático de filosofía en dicha universidad. Su continuador fue Hajime Tanabe (1885-1962) sucesor en la cátedra de Nishida y, a nuestro parecer, alcanzó su máximo esplendor con su tercer gran representante, Keiji Nishitani (1900-1990). Es a partir de estos tres pensadores -Nishida, Tanabe y Nishitani- cuando la escuela de Kyoto se consolida y proyecta como movimiento de ideas filosóficas, religiosas e incluso sociopolíticas. En su entorno se sitúan cierto número de figuras secundarias, algunas de ellas con un interés restringido únicamente al ámbito de la filosofía. La escuela de Kyoto tras la Segunda Guerra Mundial sufrió un oscurecimiento y su resurgimiento, ya en la década de los ochenta del pasado siglo, fue estimulado por filósofos y teólogos occidentales que, al disponer de textos traducidos, pudieron apreciar con nitidez la relevancia de su filosofía, con mucha más claridad incluso que los especialistas japoneses en historia de las ideas. Este acontecer tuvo sus efectos en el propio Japón y contó con el apoyo de que por entonces Nishitani aún estuviese vivo y en activo pues continuó reci- 
biendo visitas de relevancia y dando conferencias prácticamente hasta su fallecimiento.

Una primera conclusión, puede que algo prematura pero hecha desde un convencimiento profundo, es que el pensamiento segregado por la escuela de Kyoto ofrece un punto de encuentro fecundo y crecientemente revitalizado para las principales corrientes filosóficas y religiosas de Oriente y Occidente. Tan sólo por ese motivo el estudio de la escuela de Kyoto es hoy fundamental para nosotros; una escuela que goza en Occidente de excelente salud, si bien todo hay que decirlo en círculos muy restringidos, y cuya influencia impregna hoy también Oriente. Se diría que su historia es la de un viaje circular que parte de Oriente, recala tardíamente en el Occidente culto donde disemina una potente semilla cuyos primeros brotes - muy sanos y fuertes- emergen en nuestro tiempo, y retorna a Oriente donde la siembra ha comenzado con interés y con un terreno abonado por la falta de un soporte teórico para asentar la convergencia inevitable y deseable de la cultura occidental y la oriental. Y la escuela de Kyoto bien puede ser ese sustrato fundamental de asentamiento entre el Oriente culturalmente budista y el Occidente culturalmente greco-cristiano; y obsérvese que lo greco-cristiano y lo budista quedan relegados en nuestro análisis a grandes marcos culturales de referencia.

\section{II}

La escuela de Kyoto es una corriente filosófica capaz de situarse a la misma altura de las corrientes las escuelas filosóficas Occidentales más fecundas e influyentes. Si Nishida fue la fuente, de eso no hay duda, él sólo no habría bastado para ubicar el pensamiento japonés en la "gran filosofía». Fue necesario el contrapunto del pensamiento Tanabe y, sobre todo la riqueza de las aportaciones creativas del pensamiento de Nishitani, a nuestro parecer el de mayor dimensión, para hablar de una nueva filosofía japonesa $y$, si se quiere, de una nueva vertiente filosófica a nivel mundial.

Vamos a procurar, en la medida de lo posible, huir de definiciones de filosofía y de tradiciones filosóficas orientales u occidentales. Así no avanzaríamos en nuestro propósito. Hay que partir del hecho de que los filósofos de Kyoto son orientales y budistas como Heidegger o Nietzsche o Witgenstein son occidentales y greco-cristianos. Pero también es un hecho cierto que ni su meta ni su contenido son orientales ni budistas. 
Como dice James W. Heising, sin duda el mejor estudioso de esta escuela en Occidente y en Oriente y profesor trashumante de Cambridge a Barcelona pasando por Estados Unidos y Méjico para echar el ancla en Nazan (Japón),

Considerar los aspectos no-cristianos y no-occidentales de sus obras como un tipo de especia japonesa, ideal para sazonar ciertas preguntas en el menú de la filosofía occidental, puede ser el modo más simple para llegar a sus ideas y también, para mantenerlas a una prudente distancia ${ }^{1}$.

Si bien la influencia de ésta escuela no está siendo todo lo amplia que su trabajo y profundidad merece, lo cierto es que, al menos sus escritos han alterado la posición de la filosofía, y especialmente de la filosofía japonesa en occidente. Por un lado han tenido y continúan teniendo un impacto considerable entre los teóricos del zen y del budismo de la Tierra Pura que, aunque sean pocos, buscan comprender mejor y de manera más abierta otras perspectivas intelectuales. Por otra parte, ayuda a hacer más accesible la filosofía Oriental a muchos occidentales que no se encuentran mentalizados para pensar en una filosofía mundial que incorpore términos tanto de Oriente como de Occidente. Porque si algo trascendente ha realizado la escuela de Kyoto es regalarnos una filosofía mundial que forma parte de nuestra herencia como lo es el pensamiento occidental con el que trabajaron y en el que hallaron inspiración, eclipsando la contribución académica que sus compatriotas especializados en nuestro pensamiento han realizado a lo largo del siglo XX.

III

Tanto Nishida como Tanabe y Nishitani compartieron cátedra en una misma universidad y, así mismo, se les identifica por compartir también presupuestos de trabajo fundamentales, Ambos aspectos son suficientes para que se les considere fundadores de una escuela sólidamente establecida lo que no quiere decir que en sus posturas filosóficas no se hallen diferencias importantes. Pero presentan aspectos comunes de los que nos ocuparemos a continuación.

Comenzaremos por decir que su aproximación a la historia intelectual de Occidente no es tan sólo filosófica sino más bien «total» ocupándose, y mucho, de otros aspectos como la religión, la ciencia y la literatura. Aproximándonos a ellos de una forma más estricta, podemos también señalar: 
No establecen una delimitación tajante entre filosofía y religión. Se trata evidentemente de una cuestión importante y difícil de condensar en pocas líneas. No obstante diremos que tratan de "tirar por elevación" y buscan alternativas de superación del problema. Para ellos la vida de la religión incluye el pensamiento filosófico como su contrapunto, como un tipo de fuerza centrífuga para sus propias tendencias centrípetas. Señalan que el budismo carece absolutamente de lo que Pablo de Tarso llama el «locura de la cruz», lo que le ha conducido por una senda diferente a la filosofía y religión occidentales. La filosofía le sirve al budismo, como principio interior de la religión y no como entorno crítico exterior. En el budismo, la filosofía no es ni especulación ni contemplación o metafísica, sino más bien una metanoia, una conversión dentro del pensamiento reflexivo que señala un regreso al yo auténtico, el no-yo del anatman. Es una filosofía que trasciende los presupuestos de la metafísica. Lo complicado es explicar esta manera religiosa de filosofar a un mundo occidental radicalizado en su manera de entender la religión, la filosofía y no digamos la metafísica; sobre todo, y no queremos ser pesimistas, cuando la posibilidad reparadora de las grandes simas abiertas entre culturas ha disminuido porque las religiones adoptan la estructura de ideologías políticas radicales y el poder identitario se exacerba y se torna violento. En este contexto, los intelectuales laicos de fondo filosófico serio, o bien reniegan de toda religión y de casi toda forma política o se alinean con posiciones político-religiosas fundamentalistas, tanto en el ámbito occidental como el oriental, básicamente por una cuestión de supervivencia. En este difícil terreno se plantea actualmente el diálogo entre culturas.

Para los filósofos de Kyoto y para nosotros, el quehacer del pensar o el trabajo intelectual profundo, supone una necesaria transformación del modo de ver las cosas de la vida. El pensar es un auto-alumbramiento, un re-nacer a un plano nuevo en el que todo se ve de manera distinta. Si tal transformación no acontece es que no se ha cumplido el fin para el cual pensamos que no es otro que el de transformarnos y sentar las bases para una transformación del entorno sobre el que ejercemos influencia. No vienen pues al caso ciertas sutilezas academicistas que discriminan o intentan discernir donde termina lo filosófico y donde empieza lo religioso. El pensar es al fin y al cabo un ver o un desocultar y ello, entre otras cosas, es la satisfacción del pensar. Si el pensamiento es parto, ascenso, cambio de lugar intelectual, inevitablemente será también una transformación de la conciencia que había sobre lo pensado, esto es sobre las cosas de la vida. Y como todo esto pertenece a las esferas de la religión, la ética, el arte, la filosofía y la ciencia, no hay porque disociarlas 
en esferas diferentes; no hay supremacía, debe haber integración y compresión. Este proyecto supera, como luego veremos, la modernidad y la Ilustración pero no para descartarlas sino para integrarlas en un proyecto más amplio y rico. También se propone superar la religión, pero tampoco para descartarla, sino para liberarla de los dogmatismos, los afanes de dominación material y "conciencial» y el absolutismo que la devienen en interesada, desconfiada y por ende cruel. La transformación de la conciencia es un tema nuclear para los filósofos de Kyoto y ello es lo que justifica las específicas tradiciones doctrinales e históricas, y no a la inversa. Por lo tanto, en la medida en que la filosofia y la religión se refieren a modos de pensamiento, los conceptos no tienen nada que perder y mucho que ganar en una co-implicación.

En el Japón no hay antagonismo entre la filosofía y la religión por un lado, y la cultura por el otro. Cultura y religión son entendidas en Kyoto como formas semejantes, como algo de cuya esencia puede hablarse con independencia de las instituciones sociales en las que es incorporada. Esta esencia abarca el sistema de valores comprensivo de valores particulares de un grupo social y, por extensión, las artes tradicionales en las que esos valores se piensan y están expresados. Como señala Heisig, el más amplio contexto sociológico y antropológico de la cultura, que incluiría la génesis, transmisión y transformación del orden social de las relaciones humanas, del trabajo, el comercio, el entretenimiento y el poder político, ha sido dejado fuera de la discusión. El resultado de una concepción, de un marco operativo mermado de conexiones entre cultura y orden social, es el de una cultura capaz, objetiva y con la fuerza y la invulnerabilidad suficiente para criticar ese orden, pero no en una cultura que en sí misma sea convertida en objeto de crítica. He aquí una concepción radicalmente opuesta a la que ofrece la cultura occidental en la que los valores tradicionales de la cultura y las estructuras sociales se han considerado, por lo general, conjuntamente, como lo es en la filosofía y en la religión de Occidente. Los filósofos de Kyoto tienden a distinguir las cuestiones del despertar religioso de las del también despertar de la conciencia social. En ello coinciden con las formas budistas tradicionales del Japón que procuran que el establecimiento de principios universales no dañe su propia cultura y partir de sus propios valores morales para forjar su crítica a la cultura y sociedad occidentales.

El yo-auténtico es el objetivo de su filosofía-religión y la persecución de tal yo-auténtico es entendido mucho más como una metáfora de su preocupación por la claridad del pensar y la transformación de la con- 
ciencia que una profesión de fe en la enseñanza budista fundamental del no-yo, coincidencia terminológica que, por cierto, no debe tomarse a la ligera.

Los filósofos de Kyoto han puesto buen cuidado en evitar cualquier confrontación con la teoría psicoanalítica o de establecer alguna relación entre el no-yo y estados psíquicos patológicos o paranormales. Heisig da una razón para ello: El budismo, tanto en China como en Japón ha carecido de la teoría simbólica tan importante en Occidente. De sus preguntas no puede deducirse nada parecido a una desmitificación radical del cosmos, o una separación de la verdad literal de aquella que es meramente simbólica. En las cuestiones de religión todo tiene un doble sentido. El psicoanálisis se centra en los estratos del significado o en las distorsiones metafóricas elaboradas por la historia particular de unas psiques particulares. La cuestión es si una mente considerada normal según los cánones de la psicología es la más indicada para "ver» a través de la terquedad y la tan traída y tan llevada autoestima, factores que eclipsan la conciencia de la realidad tal como en verdad es dicha realidad. Los filósofos de Kyoto abordan la misma cuestión pero desde la perspectiva opuesta. En vez de considerar las distorsiones que en cada individuo pueden aparecer acerca de cómo es su percepción del mundo, buscan una vía alternativa que contempla los desperfectos generales que son parte de nuestro destino común en tanto que somos humanos. Y a la inversa, si consideramos toda percepción como un acto simbólico, hasta las percepciones consideradas como mecánicamente más fiables y aquellas que revelarían hechos objetivos no son sino factores de distracción del verdadero conocimiento del mundo, que nos inducen a manipularlo, y nos apartan del verdadero despertar al mundo tal cual es en realidad, sin la interferencia de la utilidad u otras preconcepciones.

Es importante señalar que la escuela de Kyoto es ajena al positivismo lógico y a la filosofía analítica en cuestiones como la transformación de la consciencia o de la iluminación filosófico-religiosa. No existe para justificar esta actitud ni una página escrita en toda la obra de los filósofos de Kyoto. Al considerar la existencia de Dios, Nishida, Tanabe y Nishitani hablan repetidamente de el, pero no de la idea de Dios tal y como puede aparecer en la filosofía y la teología de Occidente, sino simplemente de Dios. Sin duda, ninguno confesó creencia alguna en un ser divino o en seres divinos en el sentido en que esos términos son usados normalmente, y mucho menos en la tradición judeo-cristiana. Tampoco se refieren a la imagen de Dios tal y como esta nos aparece en las religiones o en la historia universal. Ni siquiera han intentado delimitar el término como sím- 
La Escuela Filosófica de Kioto como paradigma...

bolo de la última realidad o como principio metafísico, ni se habla de una realidad ontológica objetiva ni de una ficción subjetiva. Este es uno de los aspectos más desconcertantes y a la vez más significativos y grandiosos de esta escuela filosófica. Del mismo modo que el no-yo funciona como una metáfora para la persecución de un estado más y más elevado de conciencia hasta alcanzar la plenitud, la idea de Dios sirve, así mismo, como metáfora que apunta a la unidad esencial de la experiencia de la conciencia con la realidad tal cual esta es, y lo hace precisamente como una idea o imagen que opera en las mentes de aquellos que creen en Dios.

Llegados a esta tesitura parecería como si Nishida, Tanabe y Nishitani hubieran pasado por alto algunas de las preguntas más serias de la historia de la filosofía, y que hubieran justificado esta carencia por razones que sólo pueden considerarse religiosas. De hecho parecen haber intentado es coger lo mejor de ambos mundos: pretenden ser religiosamente budistas cuando una crítica filosófica les crea algún tipo de conflicto, y a la vez están siendo filósofos al estilo occidental cuando las objeciones serias provienen del campo budista ortodoxo, que por otra parte son harto escasas.

El punto crucial de su filosofía, lo que la ensalza hasta la cumbre del pensamiento mundial, es su originalísima persecución de la transformación de la conciencia, hecho en el que han centrado sus esfuerzos, ofreciendo un punto de vista suficientemente coherente, capaz simultáneamente de realzar esas áreas de la filosofía perenne que tratan el mismo tipo de pregunta, y de revitalizar el mundo cerrado de su propia tradición intelectual por medio del valor y calado de su propia capacidad filosófica.

\section{IV}

Hay que decir que todavía carecemos de un conocimiento preciso y ordenado que relacione a las tres figuras fundamentales que analizamos en este artículo. Esa carencia de conocimiento se refiere tanto al desarrollo histórico de sus ideas como en términos de filosofía comparada. Incluso hoy se discute el peso específico de cada uno, quizá ignorando que en realidad fueron esencialmente complementarios e incluso críticos entre ellos. Pero hay un rasgo importantísimo que les caracteriza a los tres y es que muchas de sus ideas hay que entenderlas como metáforas de las ambigüedades que señalaron la entrada del Japón en el mundo moderno porque, si se miran bien sus textos, existe una amplia trama de conexiones entre sus pensamientos y los cambios históricos, la mayoría inconscientes para ellos mismos, pero evidentes para nosotros. Heisig, la máxi- 
ma autoridad viva a la hora de considerar esta escuela es muy tajante en sostener esta afirmación. Y ello es importante porque la pretensión de posibles diálogos entre distintos culturas pasa, no por el juicio del otro sino por su comprensión que en nuestro caso es intento de comprensión teórica de las formulaciones del otro ya sea musulmán, hinduista, taoista, budista, animista o practique un aparentemente exótico sincretismo. A la masa fanatizada por mediadores interesados es fácil manejarla. Lo que se impone es el análisis de las ideas del otro allí donde puede realmente haberlas, y a partir de su.conocimiento profundo que excluye los bandazos políticos temporales, ir encajando el puzzle de su civilización con la nuestra en un verdadero diálogo respetando las formalidades de la ética del discurso propuestas en occidente por Habermas.

Cierto es que en occidente vivimos tiempos de decadencia, tanto de la modernidad como fuente de pensamiento y acción, como de la religión en su conjunto. La emergencia de la psicología de la religión, la llegada, no siempre a través de las mejores manos, de las espiritualidades orientales y el vacío espiritual acaecido en las sociedades industriales y postindustriales forman parte de un fenómeno más amplio: la pérdida de autoridad de los guardianes de la tradición y su transferencia a un sacerdocio nuevo de expertos, cuyo paradigma es en el mejor de los casos la ciencia y en el peor y más amplio, el dinero que domina las masas mediante la cultura del espectáculo bien sea este deportivo, musical o relacionado con la televisión, la moda o la imagen. Ni Japón lo estuvo, ni ahora China, India, Indonesia, los paises del suroeste asiático, Pakistán Irán, o determinadas ex-repúblicas soviéticas, están exentas de este clima y este es peligroso porque resulta imprevisible. Porque frente a el por un lado pueden surgir poderes identitarios muy graves que conviertan esos países emergentes en potencias agresivas y, por el otro, ellos mismos pueden enzarzarse en luchas internas que los desangren y desgarren.

Cuando la filosofía generada en Kyoto iba tomando forma, en Occidente se producía un cambio considerable en el panorama filosófico. Incluso las filosofías continentales, preocupadas por otro tipo de preguntas, iban en otra dirección. Al emerger al otro lado de Wittgenstein, Foucault, Derrida, Habermas y Gadamer, la escuela de Kyoto podría parecer un anacronismo. Es más, no pocas ideas sobre las que trabajaron Nishida, Tanabe y Nishitani han sido dejadas de lado por los nuevos avances de la ciencia. 


\section{La Escuela Filosófica de Kioto como paradigma...}

Sin embargo, ya hemos repetido varias veces que la escuela de Kyoto ocupa por derecho propio un lugar en la filosofía mundial. Y esta aseveración necesita de una justificación.

En primer lugar, hay una cuestión sobre la idea del no-yo como sujeto de la elección moral. Cualquier fragmento de la historia religiosa e intelectual de Oriente en el que se haya destacado la idea del no-yo demostrará que esta ha sido siempre polivalente. $\mathrm{Al}$ actuar como principio orientador de la meditación o de la práctica ascética, donde halla su pleno sentido una liberación del yo ordinario, la idea del no-yo puede funcionar sin que ello implique consecuencias inmediatas para la moral cotidiana. Sensu contrario, visto como ideal de una bondad humana básica en la vida cotidiana, la idea del no-yo puede entenderse como una llamada permanente, un recordatorio del mandato moral contra el egoísmo en las relaciones personales y sociales, sin que se oriente hacia la negación radical del yo central en la meditación y la ascesis. Y cuando la idea se sitúa en el campo de la epistemología, adquiere dimensiones que no requieren conexión alguna con la práctica de liberación del yo ni con la moral de actuar desinteresadamente en relación con los otros, aunque ambas puedan funcionar perfectamente sin llegar a ninguna decisión sobre la naturaleza de la subjetividad o la estructura última de la realidad. Al observar como la idea del no-yo obra realmente desde estos lugares diferentes, lejos de inducir a confusión, favorece incluso la comprensión del no-yo.

Los filósofos de Kyoto no se aproximaron a la idea del no-yo de esta forma. Más bien, buscaron el mismo tipo significado singular y unívoco que la filosofía occidental siempre ha atribuido a la noción de sujeto. El sujeto de la experiencia y la intuición artística no podía ser descrito de una manera muy diferente a como es descrito el sujeto de la reflexión y el conocimiento, claro está. Para nuestros filósofos, ello condujo a un nivel más elevado de comprensión, y no a una aproximación fenomenológica u operacional.

El hecho es que en su pensamiento le fue negado su lugar a la vertiente moral del no-yo, lo que entorpeció a su cometido de llegar a ser un significado unívoco y comprensivo. Como hemos visto, simplemente absorbieron la dimensión moral en su idea genérica del sujeto, reduciendo la práctica de la virtud, junto con la noción de voluntad práctica, a cuestiones de experiencia y conocimiento. Ninguno de ellos proporciona una definición clara, precisa y univoca del no-yo y de sus correlativos el yo verdadero y el ṇo-ego que ellos mismos decían que era necesaria. El resultado viene a oscurecer ese estrato moral del significado que histórica- 
mente ha sido nuclear a la idea del no-yo. Creemos que todo esto no es solo un vacío o carencia en el pensamiento de la escuela de Kyoto que hay que complementar, sino una pregunta clave en el encuentro de las filosofías orientales y occidentales.

En segundo lugar, debemos abordar la relación existente entre la auto conciencia y la crítica de la concepción antropocéntrica de la realidad. El desafío al modelo sujeto-objeto como una imposición que oscurece más la naturaleza de la realidad de lo que se supone es capaz de iluminarla, es algo crucial en el pensamiento de Nishida, Tanabe y Nishitani. De alguna manera, podría entenderse que la totalidad de su pensamiento no es sino un experimento que busca remplazar ese modelo; ello les lleva siempre de una manera uniforme y reiterativa a confrontar -quizás sería mejor decir forcejear- con cada uno de los pensadores tradicionales que analizan. La lógica del locus, la lógica de lo específico y el punto de vista de la vacuidad dependen de la crítica que realizan a cómo la realidad es distorsionada para que se adecue a la idea de la conciencia centrada en el sujeto. En este proceso, van seleccionando ideas similares de la filosofía clásica, generalmente de pensadores místicos, o bien del mundo del arte y la literatura que tratan de «mudarse» de la periferia hacia el centro.

Para ellos la estructura de la realidad es algo así como un auto despertar sin sujeto. El progreso del individuo hacia el despertar se contempla y se mide en términos de su liberación del egocentrismo y su resignación de las cosas, tal como son ${ }^{2}$. Además, se insiste, como en otras modernas tradiciones de sabiduría que giran entorno a Ken Wilber ${ }^{3}$, que el «sujeto» no-yo de este proceso trasciende al individuo y revela el obrar de la realidad. Lo real es mucho mas real cuando alcanza la conciencia, una conciencia en su forma más pura pero conciencia al fin al cabo. Los filósofos de Kyoto presuponen que no es posible una autoconciencia en el mundo inanimado, ni siquiera en el mundo de los seres sensibles no humanos, de manera que lo humano está más firmemente ubicado en el centro de la realidad que lo que pueda estar en el modelo sujeto-objeto4.

El problema que acabamos de plantear, nos conduce a una cuestión importante y no resuelta que la filosofía segregada por la escuela de Kyoto puede expresar mejor que ninguna otra filosofía en Occidente: los límites de la superación del antropocentrismo en la conciencia. Si el no-yo es la medida para el ideal del estadío más radicalmente desinteresado, liberado y despertado que el individuo humano puede lograr, entonces el valor de este estadío precisa ser aclarado en relación con otros estadíos de existencia. De otra forma, no hay manera de evaluar lo que merece ser sacrificado para lograrlo y a qué valores mayores merecería sacrificarse 
el despertar mismo. Nosotros suponemos y la escuela de Kyoto también, que la filosofía es, ante todo, un tratar de despertar al individuo humano. Ello nos obliga a preguntarnos sobre el valor de la filosofía para elucidar el resto de la realidad. Antes de llegar a la implicación moral que supone la importancia de la supervivencia y bienestar humano en el esquema más amplio de la realidad, la cuestión de si y en qué medida el presupuesto antropocéntrico es una condición necesaria para conocer la realidad, es algo que tiene que ser articulado mucho más nítidamente de lo que lo ha sido hasta ahora.

La tercera cuestión se refiere a un legado importante de los tres filósofos de Kyoto y tiene que ver con una noción radicalmente despersonalizada de Dios. Al revisar la forma en que Nishida, Tanabe y Nishitani contemplan a Dios en sus textos, observamos que emergen dos ideas distintas que se usan indistintamente con el mismo término lingüístico. Por un lado, está la idea cristiana de un Dios que pertenece irrevocablemente al ser y, por consiguiente, cuyo carácter absoluto debe verse como relativo al verdadero carácter absoluto que es la nada. Por otro lado está su idea reformulada de Dios como imagen de la nada, cuyo reconocimiento está enredado a causa de presupuestos doctrinales pero que se hace transparente al filósofo que no comparta estos presupuestos ni parta ni parta de una definición de la realidad aferrada a la noción de ser. Estas dos ideas se cruzan en el camino de su filosofar cuando se vinculan cuando se vinculan a un teología kenótica ${ }^{5}$ del «autovaciarse» que sugiere una idea cristiana de de Dios como no-yo que se acerca a la noción de la nada absoluta. Esta encrucijada de ideas llega hoy con más facilidad a los occidentales que cuando fue propuesta por los filósofos de Kyoto, mediado el siglo XX. La filosofía de la religión contemporánea y muchos teólogos también, han roto con la idea de que la búsqueda de la verdad en la tradición bíblica requiere una interpretación literal de las ideas fundamentales, incluso la idea de Dios. El acercamiento a las verdades religiosas como símbolos que señalan impulsos básicos e intangibles en nuestra naturaleza humana común, como experiencias particulares que caen fuera de los patrones normales de relaciones con el mundo o con otras personas, o como alguna forma de tarea moral o intelectual que ha de ser cumplida o apropiada por el individuo para que se haga "verdadera", han acabado por debilitar la conexión, hasta hace bien poco indiscutible, entre Dios y el ser.

La ambigüedad que los filósofos de Kyoto manifiestan en sus ideas paralelas de Dios -sus ideas propias y la cristiana tradicional- significa que de hecho ha llegado a ser una cuestión también para Occidente, y que 
la posibilidad de transferir el peso de la idea de «Dios existe» a «Dios es nada" no es ya tan descabellada como en principio podía parecer, Y precisamente porque significa esto, también significa que la noción de Dios, metafísica o simbólicamente hablando, puede servir de punto focal para atraer críticas por parte de la filosofía del ser directamente hacia la pregunta de la suficiencia una filosofía pura para la nada.

\section{VI}

Las tres cuestiones que acabamos de abordar sobre el no-yo como sujeto moral, los limites del antropocentrismo y la desvinculación de la idea del ser, han penetrado muy poco en las corrientes dominantes de la filosofía occidental. Sin embargo tienen la virtud de estar entre las muchas ideas provenientes de la escuela de Kyoto que han estimulado el encuentro y la discusión serena entre estudiosos budistas y cristianos particularmente en Japón. Ya que esta ha sido una de las vías principales para que el pensamiento de esta escuela gane terreno y atención, creemos que merece la pena considerar los factores que la han hecho posible.

En primer lugar, hay que decir que ni Nishida ni Tanabe tomaron parte entre discusiones formales entre budistas y cristianos, ni promovieron directamente sus ideas religiosas para animar tales encuentros. Para decirlo todo, este dialogo era entonces desconocido e impensable. Es mas, ambos se habrían sentido incómodos de representar a las dos tradiciones o de mediar en un encuentro entre ellas desde la posición neutral de la filosofía. No podemos concluir que aquello que Nishida y Tanabe ofrecen sea una religión mas allá de la religión; tampoco el dialogo interreligioso ofrece tal perspectiva. Además, la idea de que personas que procesan distintas clases de fe pudiesen alejarse de las formulaciones doctrínales tradicionales para abordarlas desde una alternativa bien religiosa o filosófica, podrían haberles parecido a Nishida y a Tanabe como algo irreligioso. Aunque es muy probable que si las circunstancias lo hubiesen permitido habrían agradecido ese diálogo, su compromiso con la filosofía era tan fuerte que ellos mismos no podían imaginar la doctrina religiosa sino en términos filosóficos.

Nishitani bastante mas moderno y todo hay que decirlo mejor estudiado por nosotros ${ }^{6}$ se comprometió mas abiertamente con el budismo y vivió lo suficiente para ver como nacía y prosperaba el dialogo budismocristianismo en Japón. Su intervención activa, que lo fue, hay que entenderla como una respuesta directa al espíritu de exclusividad y autori- 
tarismo que por mucho tiempo había lastrado y bloqueado la proyección de ambas religiones. Ha de pensarse que en todo caso las ideas de la escuela de Kyoto calaron hondo en el Japón y que Nishitani fue a la vez un factor de dinamización y de atracción para los círculos académicos más serios.

Desde el S. XVII, cuando Leibniz y otros comenzaron a interesarse por los primeros informes sobre el pensamiento confuciano enviadas a Europa por misioneros Jesuitas, el mundo occidental tendió -y aun muchos mantienen esta posición- a considerar estas tradiciones como esotéricas a nuestro mundo occidental. Solo una élite cultural e intelectual se interesó por estas cuestiones: filósofos como Schopenhauer o Nietzsche consideraron que la filosofía oriental tenía un valor intrínseco y una gran profundidad. Todo cambió en el S. XX en el que las tradiciones orientales están ya en la academia, en los libros de filosofía y en los libros de historia filosófica. No obstante, a estas alturas no podemos decir que hayan sido aceptadas por el pensamiento filosófico y teológico dominantes y tampoco penetrado profundamente en la educación general de las humanidades. Además, la vectorialización misional se ha equilibrado cuando no desplazado su equilibrio hacia occidente que es ahora territorio misionero para todas las religiones orientales. Con toda la cautela que procede, hemos de decir que estamos cada vez mas convencidos de que existen muchos indicios que demuestran que Occidente está al borde de una gran revolución en su espiritualidad tradicional, y que en esa revolución la sabiduría oriental puede actuar como catalizador o como elemento muy influyente. Entre tanto, el abanico de respuestas por parte de la intelectualidad oficial, tanto filosófica como teológica, se extiende desde las posiciones más superficiales u obsoletas, hasta las serias, pero lo cierto es que no parece tener el mas mínimo poder para desacelerar un proceso que se intuye ya próximo. La incursión manifiesta del pensamiento oriental en Europa y América ha tenido lugar simultáneamente al descubrimiento de que en Japón existía una filosofía global de sustrato occidental. Cuando Occidente comenzó a abrirse al dialogo con oriente los filósofos de Kyoto ya habían producido suficiente literatura basada en la interpenetración de los dos mundos. Y lo hicieron de manera muy diferente a cualquier vía producida por los estudios religiosos en occidente. La escuela de Kyoto no plantea a occidente un desafío porque el discurso lo realiza en el plano religioso y no en el teológico. Para nuestros filósofos, carece de sentido una confrontación entre fe y razón y el mencionado discurso, si se dispone de una mente lo suficientemente abierta, es valido tanto para el individuo budista o cristiano como para el agnóstico. No 
es un diálogo interreligioso ordinario en el que haya que negociar o imponer la supremacía de unas "verdades» sobre otras. Es un regalo que se nos ofrece gratuitamente para abrir nuestra conciencia a la posibilidad de abrir los ojos a un horizonte mucho más amplio que aquel en que parece haberse auto restringido la cultura dominante.

\section{VII}

En principio no hay distinción entre filosofía y religión en la escuela de Kyoto, al menos como la que se establece en occidente. El propio Tanabe se resistía al principio a la fusión de las dos, pero luego llegó a estrecharlas con más entusiasmo que Nishida o Nishitani. Gershom Scholem ${ }^{7}$ ha reiterado en que no hay «la mística» más allá de la tradición, sino tan solo una mística judía, una mística cristiana, una mística islámica etc. Esta afirmación que no compartimos y que nos sería fácil rebatir, da pie sin embargo a otra de Heisig con la que si estamos de acuerdo: se puede decir que realmente no hay ninguna cosa que pueda llamarse filosofía o religión libre del lenguaje la imagineria y los significados culturales que cada una usa para expresarse ${ }^{8}$. Por lo tanto, cuando decimos que los filósofos de Kyoto han suprimido las líneas de demarcación entre filosofía y religión esto no debe ser entendido como algo hecho dentro de un contexto occidental, sino en un marco de discurso nuevo, diferente, infinitamente más amplio. Hay quien puede caer en la tentación de decir que Hegel y Jaspers ya habían incluido la religión en la filosofía, pero su proceder no puede ser comparado directamente con la estrategia de los filósofos de la escuela de Kyoto. Filosofar sobre religión tiene un significado en el contexto judeo-cristiano y otro en el budista y ambos difieren de lo que se ha dado en llamar "estudio científico de la religión». Para Nishida, Tanabe y Nishitani, el sustrato primario o materia prima para la coincidencia entre filosofía y religión es siempre budista, y más específicamente un budismo enfocado en la búsqueda del autodespertar. Su trabajo no pretendió armonizar desacuerdos proposicionales entre diferentes tradiciones religiosas desde una posición neutral y objetiva ni usar un conjunto de «verdades» para criticar otro. El objeto de su atención -la realidad y el lugar de la conciencia en ella- no estaba vinculado a ninguna cultura especifica o historia intelectual, pero su atención si lo estaba.

Esta distinción es importante pero difícil de sostener en la practica al aplicar ideas filosóficas occidentales mediante una manera de pensar característicamente japonesa a lo que consideramos más universal en la 
naturaleza humana; su objetivo era ver lo que esa perspectiva era capaz de revelar y también de encubrir. Así se liberaron de los modos tradicionales del pensar japonés, no suprimiéndolos, sino realzándolos y amplificándolos. Como hemos dicho a lo largo de todo este artículo, su intención siempre fue introducir la filosofía japonesa en la mundial, usando como vehículo la filosofía oriental y así ofrecer una nueva perspectiva al modo de pensar japonés y por ende oriental, atrapado en la fascinación de su propia unicidad.

Este es el sistema dentro del que tratan de criticar y adaptar la tradición cristiana. Claro está que su lectura sobre esta religión se centró siempre en filósofos y místicos con lo que perdieron algunos matices fundamentales. Nunca hemos pretendido decir que Nishida, Tanabe y Nishitani pretendan haber descubierto una filosofía mas allá de la historia de la filosofía o una religión mas allá de la historia de la religión. Por eso, el problema de cómo acercarnos a la amalgama original de filosofía y religión que encontramos en la escuela de Kyoto se halla íntimamente relacionado con su propósito de abrir una ruta entre Oriente y Occidente. Desafortunadamente, resulta mucho más fácil criticar los resultados que emular el proceso.

El occidente sobre el que escriben es altamente selectivo, centrado en la filosofia oriental que va de Descartes a Heidegger, atendiendo también al arte y la literatura de este periodo. Pero la cultura viva de occidente dentro de la cual esta historia tomó forma, está ausente.

Tanto el «Oriente» como el «Occidente» de los filósofos de Kyoto tiene una parte de invención. Como estos pensadores sabián los esfuerzos que ejercieron en las zonas fronterizas de la filosofía y la religión para construir un puente que uniese la brecha entre Oriente y Occidente, no pudieron competir con lo que lograban la ciencia y la tecnología casi de manera automá tica y, sobre todo, con mucho menos esfuerzo consciente. Su crítica de la ciencia se fundamenta especialmente en que la preocupación por los resul tados implicó muchas veces un tipo de autoengaño. Desafortunadamente, la divulgación del método científico propiamente occidental no ha sido un he cho transcultural, transrreligioso y transfilosófico sino tan colonial en su es tructura como lo había sido la modernidad misma. La ciencia y la tecnología no nos acercan a los sentimientos íntimos de los pueblos orientales sino que los enmascaran. Y en ese enmascaramiento, en ese no saber que sucede en el trasfondo de las conciencias, en esa no comprensión de los recovecos más íntimos de su cultura, radica el mayor riesgo para occidente.

Para finalizar, creemos que los filósofos de la escuela de Kyoto han proporcionado a occidente un camino hacia oriente como ningún otro. El suyo 
no es un pensamiento oriental diluido para el consumo extranjero, ni es tampoco una simple transferencia que supone un fondo de conocimiento de la historia de las ideas orientales. Hace una contribución no solicitada a la filosofía mundial que respeta las tradiciones de la filosofía tanto como las expande. Nunca Occidente ha producido un movimiento intelectual cuya contribución a Oriente pueda compararse con lo que nuestros tres filósofos nos han ofrecido a los occidentales. Al hablar de procurar una aproximación entre civilizaciones, y buscar una comprensión mutua y reciproca y porque no, un acercamiento en un marco de respeto mutuo, habrá que profundizar en las ideas y considerar que los vaqueros y la coca-cola pronto se verán desbordados por otros instrumentos de consumo de corte oriental mucho mas atractivos e ingeniosos que aquellos que nosotros podemos ofrecer. El dialogo con oriente debe hacerse sobre bases profundas, por los senderos que han transitado los sabios y no por las rutas que se han movido con excesiva torpeza los políticos mirando siempre el interés a corto plazo. Nosotros tenemos a mano la ética del discurso para que ellos se sientan cómodos pero prescindamos de patrioterías, altanerías y supuestas superioridades con quien por lo menos nos iguala en cultura y sabiduría.

\section{Bibliografia}

ARCO, J. DEL: Del nihilismo a la vacuidad: Keiji Nishitani, Revista Debats, $\mathrm{n}^{\circ} 82$ Valencia, otoño 2003.

Berque, Augustin, ed.: Logique du lieu et dépassemente de la modernité. Vol. 1, Nishida: la mouvance philosophique, Ed. Ousia, Paris, 2000.

Bowers, Russell H. JR.: Someone or Nothing Nishitani's «Religión and Nothingness" as a Foundation for Chistian-Buddhist Dialogue, Peter Lang, New York, 1995.

CARTER, RoBert E.: The Nothingness beyond God: An introduction to the Philosophy of Nishida Kitaro, Paragon House, St. Paul, 1997.

DALlmaYR, F.: Nothingness and Sunyata: A Comparison of Heidegger and Nishitani, Philosophy East and West 42/1:37-48.

Frank, F., ed.: The Buddha Eye: An Anthology of the Kyoto School, Crossroad, New York, 1982.

JAMES, F.: Alterity in the Thought of Tanabe Hajime and Karl Ranher, Ph.D. dissertation, University of Chicago. Ann Arbor: University microfilms, 1988.

JAMES, F.: Cosmology and Metanoia: Buddhist Path of Process Thought for the West. The Eastern Buddhist 22/1: 111-27.

GonZÁlez Valles, J.: Historia de la filosofía japonesa, Tecnos, Madrid, 2000.

HeIsIG, J.: Sunyata and Kénosis. Academia 43: 1-29. Reprinted: Spirituality Today 39/2 (1987), 132-42; 39/3: 211-24.

HeISIG, J.: The Religious Philosophy of the Kyoto School. UNNO y HEISIG, 1990.

HeIsIG, J.: The "Self that is Not-a-Self». Tanabe's Dialectics of Self-Awareness. UNNO y HESIG, 1990, 277-90. 


\section{La Escuela Filosófica de Kioto como paradigma...}

HeISIG, J.: Philosophy as Spirituality: The Way of Kyoto School. Takeuchi Yoshinori, ed., Buddhist Spirituality. Volume 2: Latter China, Korea Japan and The Modern World, Crossroad, 367-88, New York, 1999.

HeIsIG, J.: Filósofos de la nada. Un ensayo sobre la escuela de Kyoto, Herder, Barcelona, 2002.

Jacinto Zavala, A., Filosofía de la transformación del mundo: introducción a la filosofía tardía de Nishida Kitaro, El Colegio de Michoacán, Méjico, 1989.

Jacinto Zavala, A.: La derecha ẹn la escuela de Kyoto. Avances de Investigación (El Colegio de Michoacán), serie 1, n 4, Méjico, 1993.

Jacinto Zavala, A.: La filosofia social de Nishida Kitaro, El Colegio de Michoacán, Méjico, 1994.

Jacinto Zavala, A.: Textos de filosofía japonesa moderna, El Colegio de Michoacán, Méjico, 1995.

Jacinto ZaVAla, A.: La otra filosofía japonesa, El Colegio de Michoacán, Méjico, 1997.

LAMBE, J.: Sur la personne et l'oeuvre de Hajime Tanabe, Revue de philosophique de Louvain 4/92: 430-58, Lovaina 1994.

MASÍA ClAVEL, J.: Filosofía del absoluto en K. Nishida: apropiación crítica de lo budista y lo cristiano por un filósofo japonés. José Gómez Caffarena y José $\mathrm{M}^{\mathrm{a}}$ Mardones, eds., Cuestiones epistemológicas: Materiales para el estudio de la religión, Anthropos, Vol. 1, 137-47, Madrid, 1992.

NisHIDA, K.: Ensayo sobre el bien, Revista de Occidente, Madrid, 1963.

NISHIDA, K.: An Inquiry into the Good, New Haven: Yale University Press [1: 1-200], 1990.

NisHIDA, K.: Indagación del bien, Gedisa, Barcelona, 1995.

NISHIDA, K.: La experiencia pura. JACINTO 1995, 67-74 [1:9-18].

NisHIDA, K.: Fundamentación filosófica de las matemáticas. JACINTO 1995, 109-52. [11:237-85].

NisHIDA, K.: Lógica del topos y cosmovisión religiosa. JACINTO 1995, 153-233. [11: 371464].

NishitanI, K.: Ontology and Utterance. Philosophy East and West 31/1: 29-43.

NisHITANI, K.: Science and Zen. FRANK 1982, 111-37. [11:227-61].

Nishitani, K.: Was ist Religion?, Franfurt: Insel Verlag. [10: 3-319], 1986.

NishitanI, K.: The Self-Overcoming of Nihilism, Albany: SUNY Press. [8:3-290].

Nishitani, K.: Nishida Kitaro, University California Press, Berkeley, 1991

NISHITANI K.: El nihilismo como existencia. JACINTO 1995, 315-27. [8: 3-16].

Nishitani, K.: La Religión y la Nada, Siruela, Madrid, 1999.

PARKES, G.: Heidegger and Asian Thougt, University of Hawaii Press, Honolulu, 1987.

PARKES, G.: Nietzsche and Nishitani on the Self-Overcoming of Nihilism. International Studies in Philosophy, 25/2: 51-60, 1993.

Stevens, B.: Histoire de l'être et nihilisme dans la perspective de l'école de Kyoto. Heidegger Studies. 12: 83-94. 1996.

Stevens, B.: Topologie du néant: Une approche de l'école de Kyoto. Editions Peeters, Paris, 2000.

Tanabe, H., Zu Hegels Lehre vom UrTeIL: Hegel-Studien 6:211-29. [3: 165-75], 1971.

TANABE, H.: Philosophy and Metanoetics, University of California Press, Berkeley, 1986.

TANABE, H.: La dialéctica de la lógica de la especie. JACINTO 1995. 247-50. [7: 253-69].

TANABE, H.: La especie como base de la estructura práxica de la lógica. JACINTO 1995, 251-81. [7:345-54]. 


\section{Javier del Arco Carabias}

TANABE, H.: El carácter religioso de la práctica. JACINTO 1995, 283-305. [7: 345-72].

TANABE, H.: Christianity, Marxism, and Japanese Buddhism: In Anticipation of a Second Religious Reformation. DILWORTH y VIGLIELMO 1998, 115-58. [10:271-324].

TANABE, H.: Religion, Theology and Philosophy: A Concluding Word on Love, Struggle and Freedom. DILWORTH y VIGLIELMO 1998, 159-89. [10: 235-69].

\section{Notas}

1 Véase Heisig James W., Filósofos de la nada. Un ensayo sobre la Escuela de Kyoto, Herder, Barcelona, 2002, p. 31.

2 Ya que esta conexión no se cuestiona nunca directamente ni tampoco se justifica, mucho del antropomorfismo del que aparatosamente se han desprendido va regresando poco a poco a medida que avanza su filosofar.

${ }^{3}$ Ken Wilber, importante pensador norteamericano contemporáneo autor de numerosas obras de vinculación y síntesis entre el pensamiento Oriental y el Occidental.

4 Véase lo dicho en la nota $\mathrm{n}^{\circ} 2$

5 Presentación débil, humillada, oculta, de Dios. Dios es amor amistoso, que se da más bien al que está dispuesto a una escucha pasiva que al que quiere apresarlo racionalmente.

${ }^{6}$ Véase Arco, J. del, Del nihilismo a la vacuidad: Keiji Nishitani, Revista Debats, $\mathrm{n}^{\circ} 82$ Valencia, otoño 2003

7 Veasé Scholem, G. Las grandes tendencias de la mística judia, Siruela, Madrid, 1994.

${ }^{8}$ Véase Heisig James W., Filósofos de la nada. Un ensayo sobre la Escuela de Kyoto, Herder, Barcelona, 2002, p. 333. 